\title{
CRISPR-Cas System: The Powerful Modulator of Accessory Genomes in Prokaryotes
}

\author{
Anca Butiuc-Keul a, Anca Farkas $^{a, b}$ Rahela Carpa ${ }^{a, b} \quad$ Dumitrana lordache ${ }^{a}$ \\ aDepartment of Molecular Biology and Biotechnology, Faculty of Biology and Geology, Babeş-Bolyai University, \\ Cluj-Napoca, Romania; ${ }^{b}$ Center of Systems Biology, Biodiversity and Bioresources, Babeş-Bolyai University, \\ Cluj-Napoca, Romania
}

\section{Keywords}

Adaptation $\cdot$ cas genes $\cdot$ CRISPR $\cdot$ Interference $\cdot$ Spacer acquisition

\begin{abstract}
Being frequently exposed to foreign nucleic acids, bacteria and archaea have developed an ingenious adaptive defense system, called CRISPR-Cas. The system is composed of the Clustered Regularly Interspaced Short Palindromic Repeats (CRISPR) array, together with CRISPR (cas)-associated genes. This system consists of a complex machinery that integrates fragments of foreign nucleic acids from viruses and mobile genetic elements (MGEs), into CRISPR arrays. The inserted segments (spacers) are transcribed and then used by cas proteins as guide RNAs for recognition and inactivation of the targets. Different types and families of CRISPR-Cas systems consist of distinct adaptation and effector modules with evolutionary trajectories, partially independent. The origin of the effector modules and the mechanism of spacer integration/deletion is far less clear. A review of the most recent data regarding the structure, ecology, and evolution of CRISPR-Cas systems and their role in the modulation of accessory genomes in prokaryotes is proposed in this article.
\end{abstract}

karger@karger.com www.karger.com/mip

Karger $\stackrel{\text { ' }}{=}$
(C) 2021 The Author(s)

Published by S. Karger AG, Basel

This is an Open Access article licensed under the Creative Common Attribution-NonCommercial-4.0 International License (CC BY-NC) (http://www.karger.com/Services/OpenAccessLicense), applicable to the online version of the article only. Usage and distribution for commercial purposes requires written permission.
The CRISPR-Cas system's impact on the physiology and ecology of prokaryotes, modulation of horizontal gene transfer events, is also discussed here. This system gained popularity after it was proposed as a tool for plant and animal embryo editing, in cancer therapy, as antimicrobial against pathogenic bacteria, and even for combating the novel coronavirus - SARS-CoV-2; thus, the newest and promising applications are reviewed as well.

(C) 2021 The Author(s)

Published by S. Karger AG, Basel

\section{Introduction}

The CRISPR-Cas system was observed for the first time in 1987 by a group of Japanese researchers [Ishino et al., 1987], even though they did not realize what this structure represents and how it works. It was only after 2005 that its role in immunity was foreseen, when was observed that many of the inserted sequences in bacterial genomes were of viral or plasmidic origin [Mojica et al., 2005; Doudna and Charpentier, 2014]. In 2012, the system was proposed as a powerful gene-editing tool [Jinek et al., 2012] and since then, the CRISPR-Cas engineered system had many applications. It was already used in 
plant genome editing [Schaeffer and Nakata, 2015; Samanta et al., 2016], treatment of neurodegenerative diseases [Kolli et al., 2018], and especially in cancer therapy [Zhen et al., 2014; Dai et al., 2019; Zhang et al., 2020]. Recently, it was proposed for combating pathogenic bacteria [Bikard and Barrangou, 2017] and human viruses [Soppe and Lebbink, 2017]. Most recent outcomes on CRISPR usage are focused on the surveillance [Kellner et al., 2019] and therapy of retroviral infections, such as coronavirus disease 2019 (COVID-19) caused by the novel coronavirus SARS-CoV-2 [Li et al., 2020; Nguyen et al., 2020].

Despite the multitude of research articles about the CRSIPR system, the detailed mechanism of action and the possible role of this immune system in the acquisition of foreign genetic material is still unknown. Thus, this article proposes a review of the most recent data regarding the structure, ecology, and evolution of CRISPR-Cas systems and their role in the modulation of accessory genomes in prokaryotes. The potential and applied impact of spacer acquisition and CRISPR array polymorphism are also discussed in this article as well as the main applications in biotechnology.

\section{CRISPR-Cas Structure}

Viruses were considered the key factors of life evolution, acting as a selective pressure on bacteria from different environments and facilitating genetic exchange. Approximately $20-40 \%$ of bacteria are killed every day by bacteriophage infection [Hampton et al., 2020]. Subsequently, it was shown that the acquisition of exogenous DNA via transduction, conjugation, and horizontal gene transfer (HGT) was followed by selection of an array of defence mechanisms in bacteria that allow the recognition of foreign DNA and distinguishing from self DNA [Horvath and Barrangou, 2010]. There are several different protection mechanisms in bacteria, and it is unclear whether clustering defence genes in islands offers a specific selective advantage [Doron et al., 2018] upon "genomic junkyards," in which the defence genes frequently acquired via HGT are accumulated, because insertion in these regions is unlikely to be deleterious [Koonin, 2018].

Thus, most archaea and many bacteria developed several autoprotection strategies against foreign nucleic acids. Among them, CRISPR arrays together with associated cas genes form the CRISPR-Cas immune system [Rath et al., 2015; Høyland-Kroghsbo et al., 2017; Jackson et al., 2017; García-Martínez et al., 2018]. Information

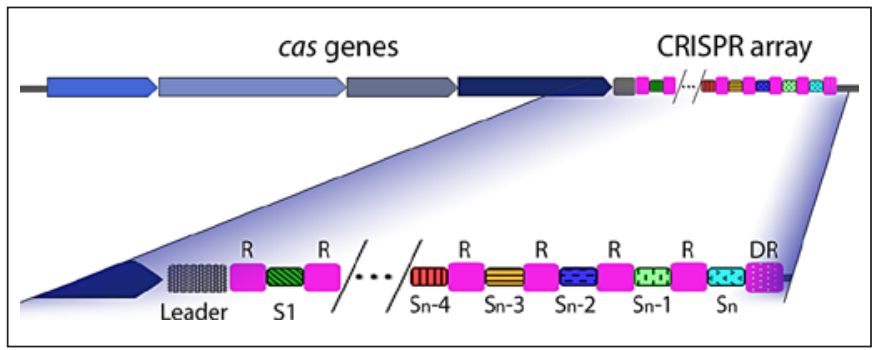

Fig. 1. The structure of a CRISPR-Cas system. R, repeat; S, spacer; $\mathrm{n}$, the total number of spacers in the array. A CRISPR array can have at least one spacer, each flanked by two repeats. The last repeat, which usually suffers mutations, is called degenerated repeat (DR).

stored within CRISPR arrays is used to direct the sequence-specific neutralization of invading genetic elements, including viruses and plasmids [Jackson et al., 2017].

The first description of the system was in 1987 [Ishino et al., 1987], and since then it has been identified in $85 \%$ of archaea and $45 \%$ of bacteria [McGinn and Marraffini, 2019]. Their localization is different, CRISPR loci can be found in chromosomes and plasmids as well [Horvath and Barrangou, 2010]. The CRISPR-Cas locus generally consists of an operon of cas genes and a CRISPR array composed of a series of conserved, sequence-specific repeats (25-35 bp long), flanking unique inserts of similar length known as spacers (26-72 bp) (as shown in Fig. 1), acquired from extrachromosomal elements [Wright et al., 2016; Jackson et al., 2017; Koonin and Makarova, 2019]. Several sequences of CRISPR spacers show homology with extrachromosomal DNA [Mojica et al., 2005; Pourcel et al., 2005], such as sequences detected in viral genomes and plasmids, called protospacers [Horvath et al., 2008]. Spacers are key elements of adaptive immunity, their acquisition during previous infections [Pourcel et al., 2005; Makarova et al., 2006] ensuring the recognition and neutralization of invaders in case of subsequent attacks [Barrangou et al., 2007].

Partially palindromic sequences were found in most of the repeats, conferring stability and highly conserved secondary structures. Palindromic repeats are predicted to generate RNAs with stable hairpin structures [Kunin et al., 2007; Koonin and Makarova, 2019]. Usually, one CRISPR locus contains conserved repeat sequences, but there is a polymorphism in the sequence and the length of the repeats between different CRISPR loci [Kunin et al., 2007]. Prior to the first repeat of each CRISPR array, there is a DNA sequence rich in adenine and thymine, called 
the leader sequence [Grissa et al., 2007]. New spacers, derived from invading MGEs are integrated into the CRISPR array through the activity of Cas proteins [Jackson et al., 2017]. The spacer sequences of a given bacterial strain, therefore, reveal the history of past CRISPR-Cas-mediated interactions. The number of CRISPR loci and the length of each locus are variable. Several bacteria contain multiple CRISPR loci (e.g., 18 CRISPR loci in Methanocaldococcus sp. FS406-22), and some of these loci can be hundreds of repeat-spacer units. The number and length of CRISPR loci are not correlated with genome size; some of the smallest microbial genomes (e.g., Nanoarchaeum equitans) contain multiple CRISPR loci [Sorek et al., 2013].

\section{Cas Proteins}

CRISPR systems consist of CRISPR arrays (spacers and repeat sequences) and adjacent CRISPR associated cas genes, which code for proteins involved in the immune response [Brouns et al., 2008] and DNA repair [Makarova et al., 2002]. Diverse cas genes were discovered in different organisms, making their classification very difficult, as the number of annotated genomes increases. At first, four cas genes were identified in genomes containing the CRISPR-Cas system [Jansen et al., 2002], but accumulating genome sequences have led to the identification of 93 cas genes grouped in $~ 45$ different gene families based on sequence similarity of the encoded proteins [Haft et al., 2005; Makarova et al., 2015]. Six of these cas genes (cas1-cas6) are widely distributed, cas 1 and cas 2 being considered as a hallmark in genomes that contain CRISPR loci [Haft et al., 2005]. The analysis of cas1 sequences suggests several distinct versions of CRISPR-Cas systems [Haft et al., 2005], each of them being defined by a unique composition and conserved arrangement of cas genes (Table 1).

The Cas proteins interact with specific sets of CRISPR loci [Makarova et al., 2006]. Different subtypes of Cas proteins were named after representative organisms that contained a single CRISPR-Cas locus (for example, Cas proteins from Escherichia coli were designated Cse1 [CRISPR system of E. coli gene1]; other subtypes included Aeropyrum [Csa], Desulfovibrio [Csd], Haloarcula [Csh], Mycobacterium [Csm], Neisseria [Csn], Thermotoga [Cst], and Yersinia [Csy]) [Haft et al., 2005]. These initial categories cannot easily handle the relationships between homologous Cas proteins, the variability of cas operons, or organisms that contain multiple CRISPR loci.
A new classification system based on the evolutionary relationships between conserved proteins and cas operon suggests the organization of the CRISPR-Cas system in two modules: the adaptation module that requires the proteins Cas1 and Cas2, which are involved in spacer acquisition, and the effector module, that is required for the processing of primary CRISPR transcripts (crRNA), interference and degradation of foreign nucleic acids. Thus, the CRISPR subtypes may differ in the Cascade proteins involved in the processing of crRNA, some CRISPR systems require multiple proteins, whereas other types require a single multifunctional protein. Multiple protein complexes were identified mostly in the class 1 CRISPRCas system, where the effector complexes of type I and type III are phylogenetically related ribonucleoproteins [Brouns et al., 2008; Makarova et al., 2015]. Unlike these, the class 2 system contains a single interference protein as it was observed in types II, V and VI [Shmakov et al., 2017]. Several other proteins containing at least one RNA recognition motif with unknown function (RAMPs) seem to be involved in the processing of pre-crRNA [Makarova et al., 2011].

\section{Classification of CRISPR-Cas Systems}

A simplified classification of these immune systems is almost impossible because most of the prokaryotes acquired multiple CRISPR loci by frequent HGT, and the CRISPR-Cas systems show a high diversity of Cas proteins. Nevertheless, 2 classes and six types with multiple subtypes of CRISPR-Cas systems were identified. Based on the distinct architecture of the effector modules, types I, II, and IV belong to class 1, and types II, V, and VI belong to class 2 [Koonin and Makarova, 2019]. Class 1 systems possess multisubunit crRNA-effector complexes, while in class 2 systems all functions of the effector complex are carried out by a single protein, such as Cas9 (Table 1). There is evidence for types IV and V, which belong to class 1 and class 2, respectively [Koonin and Makarova 2019]. There are several differences between the CRISPR-Cas systems in terms of expression, interference, and adaptation modules [Makarova et al., 2015]. Although most of the prokaryotes contain one type of CRISPR-Cas system, the coexistence of different types of systems was also reported. Class 1 CRISPRCas systems are considered evolutionary ancestral systems. The class 2 systems have evolved from class 1 systems via the insertion of transposable elements encoding various nucleases, and are now being used as tools for genome editing [Mohanraju et al., 2016]. 
Table 1. Major Cas proteins (other Cas proteins are reviewed by Makarova et al., 2011)

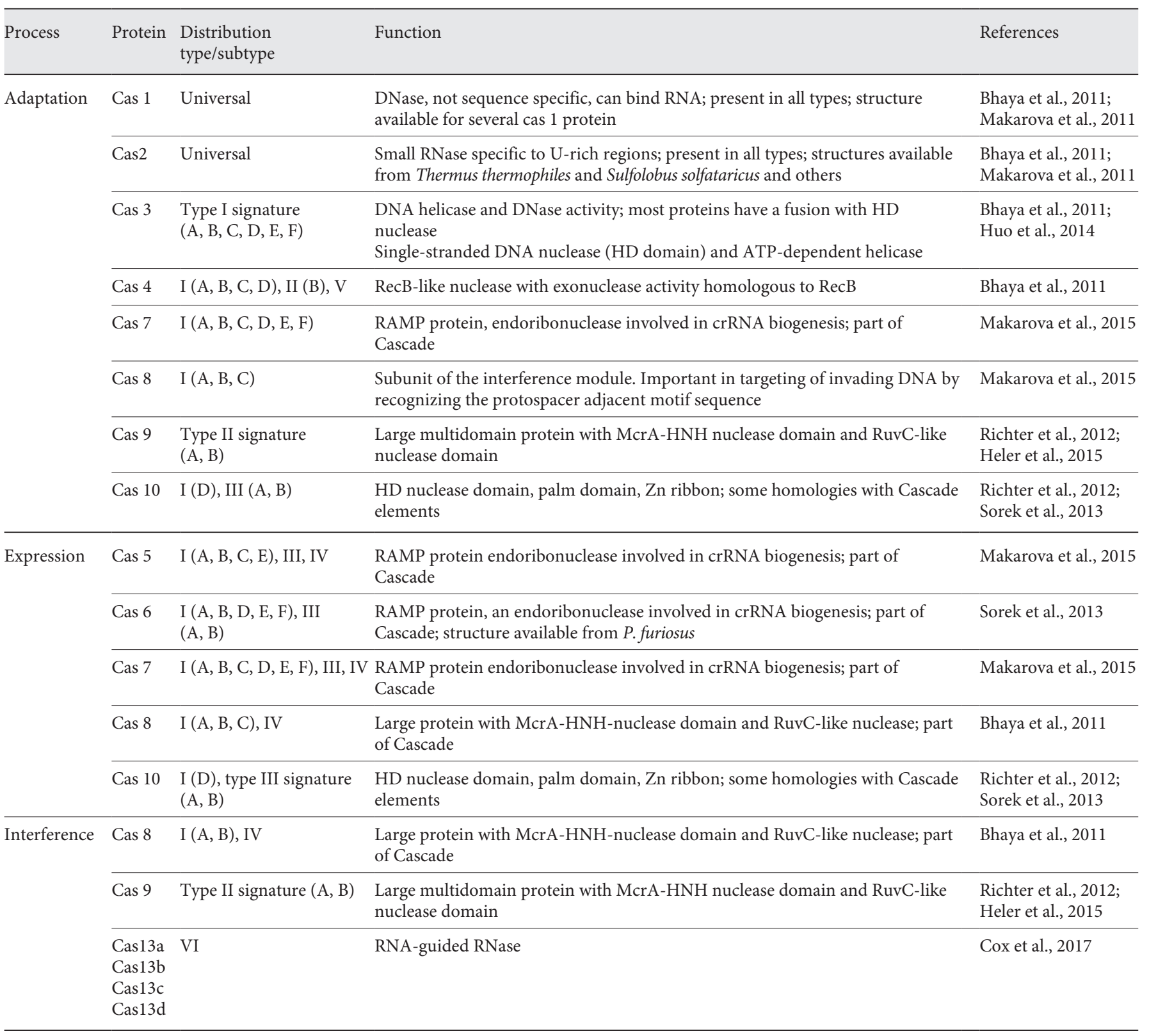

The common feature of all type I systems is the presence of protein Cas3 [Sinkunas et al., 2011]. The number of cas genes is variable and defines six subtypes of the type I system (type I-A to type I-F). All type I systems encode the Cas1, Cas2, Cas3 proteins as a Cascade-like complex. Cascade complex is involved in target localization, spacer acquisition, and crRNA processing. The type I-A systems show a particular Cascade complex with Cas3 as part of the complex [Rath et al., 2015]. The Cas1, Cas2, Cas9 proteins, and sometimes Csn2 or Cas4 are encoded by type
II CRISPR-Cas systems [Barrangou et al., 2007; Heler et al., 2015; Wei et al., 2015]. Cas9 is involved in adaptation, crRNA processing, and cleavage of the target DNA [Heler et al., 2015; Wei et al., 2015]. Type II systems were further divided in subtypes II-A, II-B and II-C. Type II-C lacks the gene for Csn2 or Cas4 [Chylinski et al., 2013; Koonin and Makarova, 2013]. The protein Cas10 was found in type III CRISPR-Cas systems, but their function is not clear yet. Most Cas proteins are part of the complexes similar to Cascade as Csm (in Type III-A) or Cmr (in 
Fig. 2. The action stages of the CRISPR-Cas system. In the first stage, adaptation, cas 1 and cas 2 genes are transcribed, and the resulting proteins form a complex that recognizes the protospacer adjacent motive (PAM) and copy the protospacer sequence. This protospacer is incorporated in the array as a new spacer between the leader sequence and the first repeat, with the duplication of the later. Alongside Cas1-Cas2 complex, other Cas proteins are involved in this stage. The second stage consists in the CRISPR array expression. The transcribed RNA (pre-crRNA), from which results crRNAs, contains only one spacer and one repeat. In the last stage, interference, the crRNA corresponding to the spacer of the invading DNA, associated with one or more Cas proteins, recognizes the specific site and cleaves both strands of DNA.

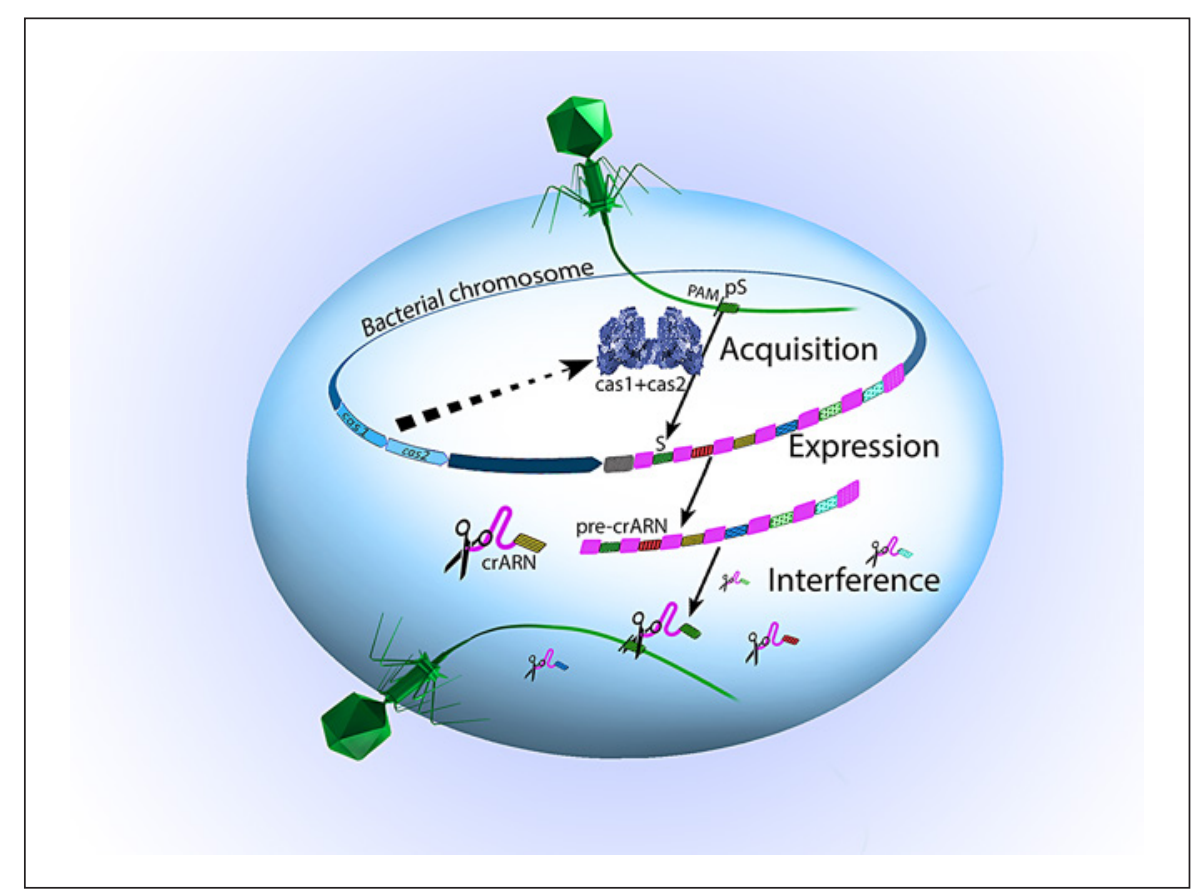

Type III-B) complexes [Rouillon et al., 2013; Staals et al., 2013].

Another difference between CRISPR-Cas systems is the target DNA in the case of type I and II systems and DNA and/or RNA in the case of type III systems. Type II system has been found only in bacteria, while the type I and type III systems in bacteria and archaea as well [Makarova et al., 2011]. Type III systems showing dual DNA/ RNA interference activity are composed of several different subunit complexes comprising multiple copies of the Cas7 and the small subunit Cas11 (Csm2 or Cmr5) [Staals et al., 2014]. Type III CRISPR-Cas system is the only system that utilizes three different nuclease activities such as specific DNA/RNA cleavage and nonspecific ssDNA cleavage to provide efficient protection against DNA and RNA invaders. The Cas7 protein might function as a switch to lower the DNAse activity of Cas10 after releasing the cleaved target RNA from the complex [Samai et al., 2015]. Type III system is flexible to mutations in the protospacer sequence [Maniv et al., 2016], being able to neutralize escape mutants from that particular system [Silas et al., 2017]. The structure and particular functions of type III system have been reviewed by Zhu et al. [2018]. Due to their particularities, different biotechnological applications based on type III systems have been developed, such as genetic manipulations, including genome engineering and gene silencing [Liu et al., 2018].

\section{Mechanism of CRISPR-Cas Adaptive Immunity}

The defence process mediated by CRISPR-Cas system can be divided into three stages: adaptation, expression, and interference (shown in Fig. 2). The first step occurs only when the cell encounters new foreign DNA for the first time. The second and third steps happen any time the cell gets infected. The sequences of invading DNA corresponding to the spacers, called protospacers, are recognized by the system due to a short conserved region within a few bases (2-5 bp) near them, known as ProtospacerAdjacent Motif (PAM) [Mojica et al., 2009; Shah et al., 2013; Gleditzsch et al., 2019]. Protospacer recognition is followed by the generation and integration of a new sequence of nucleic acids identical to the protospacer into the CRISPR array as a new spacer, followed by reparation of the CRISPR array by cellular repair proteins [Wang et al., 2015; Hille and Charpentier, 2016], and the duplication of the proximal repeat [Liu et al., 2017]. Spacer acquisition from RNA (transcripts of a DNA genome of a MGE via reverse transcription) is possible by a reverse transcriptase mostly fused to the Cas1 protein [Silas et al., 2016, 2017].

Many of the CRISPR-Cas systems have highly consistent system-specific spacer lengths, except the type III CRISPR with variable length of spacers. The PAM sequence within the pre-spacer substrate allows the correct integration of new spacers into the CRISPR array, to 
avoid the alignment at the wrong end of the crRNA target binding site. This process is mediated by Cas1-Cas 2 complex. The Cas 1 protein is involved in the cleavage of the CRISPR array at the leader-repeat junction and joining of the incoming spacer in-between of the repeat strands, the nicking and ligation occur in a concerted manner, corresponding to a classical integrase reaction [Arslan et al., 2014]. Such a mechanism is consistent with the predicted integrase activity of the Cas1 protein [Makarova et al., 2006]. Some authors consider that the integration of new spacers consists in two cleavages at the insertion site. The first cut, mediated by Cas1 or Cas2, occurs at the CRISPR/ leader boundary, while the second nick occurs at the leader-distal end of the CRISPR, being determined by the distance from the previous cleavage. Cas1 protein interacts with Cas2 protein to form a complex that acts as a spacer integrase. This heterohexameric complex (Cas1-Cas2) contains two separate DNA-binding regions, one that binds the incoming protospacer and one that binds the CRISPR array. Once loaded with the incoming spacer, the Cas1-Cas 2 complex catalyzes two cleavage-ligation reactions, first at the leader end of the first repeat, and subsequently at the spacer end of the repeat [McGinn and Marraffini, 2019]. Acquisition process also requires non-Cas proteins involved in DNA repair by the interaction of Cas1 with key components of repair systems such as RecB, RecC, and RuvB [Babu et al., 2011]. This mechanism was confirmed by Díez-Villaseñor et al. [2013]. Details about the function of Cascade complex are reviewed by Jackson et al. [2017].

Another important aspect is the acquisition of spacers from foreign DNA, and not from host DNA, to avoid the mechanism of self-targeting, which is similar to autoimmunity in eukaryotic adaptive immune systems [Stern et al., 2010]. Acquisition of spacers from MGEs, other than those from host CRISPRs is termed naïve CRISPR adaptation and requires pre-spacer substrates generated from foreign material [Fineran and Charpentier, 2012]. Further studies are required to clarify how the diverse CRISPR-Cas systems balance the requirement for naïve production of pre-spacers from MGEs against the risk of acquiring spacers from host DNA. It is already known that in type II systems, spacer acquisition appears biased towards MGEs, and nuclease-deficient Cas9 fails to discriminate between host and foreign DNA [Wei et al., 2015].

In the second stage, the spacer is transcribed into a precursor of CRISPR RNA (pre-crRNA) that is subsequently processed into mature crRNA. The expressed spacer sequence provided by the crRNA is thought to rec-

CRISPR-Cas: The Immunomodulatory

System in Prokaryotes ognize and guide the complex to bind the specific protospacer target. At the expression-processing stage, the precrRNA is processed to generate mature crRNAs by a distinct complex of Cas proteins, a dedicated processing nuclease (Cas6), a single large Cas protein or an external RNase [Brouns et al., 2008; Sorek et al., 2013; Rath et al., 2015; Mohanraju et al., 2016; Barrangou and Horvath, 2017].

In the third stage, interference, the foreign nucleic acid is recognized and destroyed by crRNA and Cas proteins. The complex of crRNA-Cas proteins locates the corresponding protospacer and triggers degradation of the target by specific Cas nucleases [Rath et al., 2015]. If there is no match between the CRISPR spacer and the foreign DNA, then the DNA sequence is not neutralized by the spacer transcript. In the case of a phage, it can reproduce inside the cell, leading to bacteria lysis and death [Han et al., 2013]. Different aspects of CRISPR-Cas biology were previously reviewed in numerous articles [Sorek et al., 2013; Charpentier et al., 2015; Mohanraju et al., 2016; Wright et al., 2016; Barrangou and Horvath, 2017; Jackson et al., 2017; Komor et al., 2017; Nishimasu and Nureki, 2017].

\section{Origin and Evolution of CRISPR-Cas Systems}

In the last decade, CRISPR-Cas systems were studied in detail, the structure and some of the biological functions of the system being deciphered [Sorek et al., 2013; Barrangou and Maraffini, 2014; Wright et al., 2016; Barrangou and Horvath, 2017]. Moreover, the Cas9, Cas12, and Cas 13 endonucleases were successfully employed as genome-editing tools [Doudna and Charpentier, 2014; Hsu et al., 2014; Komor et al., 2017; Wu et al., 2018]. Being a defence mechanism, rapid evolution of the sequence of some of the cas genes in the effector module [Takeuchi et al., 2012] and diversification of the organization of the CRISPR-Cas loci were observed [Makarova et al., 2015; Kooninetal., 2017]. Despite the unclear origin of the effector modules, most of them were acquired by several classes of MGEs [Makarova et al., 2015; Mohanraju et al., 2016; Koonin and Makarova, 2019] and the components of CRISPR-Cas systems were recruited by various MGEs as well. Transposable elements similar to Tn7 encode different variants of CRISPR-Cas [Faure et al., 2019]. The adaptation process and the structure and function of the effector modules of the CRISPR-Cas systems are not completely elucidated due to their high diversity and autonomy. Usually, the adaptation module remains fixed, 
while the effector module is changed in different variants of the same CRISPR-Cas type or subtype [Hudaiberdiev et al., 2017; Koonin and Makarova, 2019]. Moreover, in numerous bacteria and archaea, stand-alone adaptation and effector modules have been found. Thus, the Cas proteins give a functional separation of the modules of the CRISPR-Cas system.

CRISPR array is usually located in the direct vicinity of cas genes [Haft et al., 2005], but in some species such as Listeria monocytogenes [Mandin et al., 2007], Aggregatibacter actinomycetemcomitans [Jorth and Whiteley, 2012], and Enterococcus faecalis [Hullahalli et al., 2015], multiple CRISPR arrays may be distant to the cas genes. Such isolated CRISPRs, called orphan CRISPR arrays, are considered as remnants of previous functional CRISPRCas systems [Makarova et al., 2015], being involved in bacterial autoimmunity [Mandin et al., 2007]. Analysis of the CRISPR arrays in the orphan CRISPRs of $A$. actinomycetemcomitans showed that spacer sequences were antisense to bacterial self-coding genes [Jorth and Whiteley, 2012], suggesting the relationship between orphan CRISPRs and the regulation of other gene expression [Stern et al., 2010]. Even if all CRISPR loci are expressed, not all of these produced crRNA trigger interference, as it was shown in Haloferax volcanii [Maier et al., 2013].

Bioinformatic analysis of cas genes and genomic cas 1 homologues showed that these proteins are not always encoded within the CRISPR-Cas loci [Makarova et al., 2013]. Cas 1 genes are found within $12-18 \mathrm{~kb}$ regions of genomic DNA flanked by terminal inverted repeats, very similar to transposable elements [Krupovic et al., 2014]. These transposable elements share two universal genes encoding Cas1, a family B DNA polymerase, and additional genes, mostly encoding diverse nucleases and DNA-binding proteins. Moreover, the mechanism of spacer integration catalyzed by Cas1 is very similar to transposon integration by integrase or casposon integration by casposase. Casposons are a newly identified group of transposons that use Cas1-like transposases for transposition. This led to the hypothesis that Cas1 could be assimilated to a casposase [Krupovic et al., 2014; Krupovic and Koonin, 2016]. Several casposons encode some virus capsid proteins, being similar to politons and are predicted to form virions [Yutin et al., 2018].

Four distinct casposon families integrated mostly into archaea and some bacteria were identified [Krupovic et al., 2017]. Family 1 of casposons were found exclusively in the archaeal phylum Thaumarchaeota and encoded PolBs of the protein-primed variety, being very similar to the corresponding proteins from archaeal viruses. The casposons from three other families encode divergent PolBs different from either protein-primed or RNAprimed PolBs [Makarova et al., 2015]. There is no evidence of the transposition of casposons, but some of the casposons are regarded as active transposons due to their mobility [Krupovic et al., 2016]. Thus, Krupovic et al. [2017], supposed that CRISPR repeats evolved directly from the preexisting casposon target site. Rollie et al. [2015] showed that Cas1, in the absence of Cas2, displays intrinsic sequence specificity to the sequence flanking the integration site. Similarly, casposase alone is sufficient to direct the specific integration of casposons into the target site [Béguin et al., 2016]. Other data strongly suggests that the leader sequence also evolved directly from the target site employed by the ancestral casposon. It was also shown that Cas1 evolved as the core enzyme of casposons and was eventually coopted to form the basis of the CRISPRCas immune system [McGinn and Marraffini, 2019]. Another key step sustaining the evolution of the CRISPRCas adaptation machinery from casposons was the formation of a pseudosymmetrical heterohexameric complex by recruitment of Cas2. There is a significant difference between DNA of casposons and protospacers. The two ends of the linear casposon DNA designated for integration are identical and can be both specifically recognized by the casposase; thus, the two termini are poised for integration at a fixed distance, but the two ends of a protospacer are not identical. The protospacer end containing the PAM-complementary sequence is recognized by one of the four Cas1 subunits, whereas the accommodation of the other protospacer terminus is dictated by the quaternary structure of the Cas1-Cas2 complex [Wang et al., 2015]. The persistence length of dsDNA is $35-50 \mathrm{~nm}$ (100-150 bp) [Mitchell et al., 2017], which precludes bending of the short 30-70 bp protospacers such that both ends of the molecule would be held by the homodimer. Thus, the transformation of the casposase into CRISPR-Cas1 requires the recruitment of Cas2 enzyme that is homologous to mRNA interference, which could have already been present in the casposon that gave rise to CRISPR-Cas adaptation machinery [Koonin and Krupovic, 2015].

\section{Role of the CRISPR-Cas System in HGT}

\section{CRISPR-Cas Mediation of HGT}

Protection against chemical (e.g., antibiotics, disinfectants, or heavy metals) and biological agents (e.g., foreign DNA structures, such as viruses) is achieved via two ap- 
parently antagonistic processes: by acquiring foreign genetic material and by limiting the intake of exogenous material. Gathered from previous infections, CRISPR spacers are heritable and store a memory of past interactions with invading genomes, but other spacers with identifiable protospacers were found to target MGEs, probably derived from species-specific microbial mobilomes [Shmakov et al., 2017]. Thus, the CRISPR-Cas adaptive immune system was supposed to be an effective defence against genome invaders such as MGEs, potentially reducing HGT, with important implications for virulence and antimicrobial resistance of pathogenic bacteria. The selective pressure of biocides (e.g., antibiotics and others), favoring the adaptation by acquisition antibiotic resistance traits was presumed to be stronger and thus prevailing over phage-induced acquisition of immunity in microbial populations [Palmer and Gilmore, 2010]. Therefore, the consumption and dissemination of antibiotics in the environment is favoring the deficient forms of immunity provided by CRISPR-Cas systems. This has been supported by studies of Palmer and Gilmore [2010] which stated that the CRISPR-Cas system is lacking in multidrug-resistant enterococci, because the system would interfere negatively with HGT. They concluded this after testing the level of coexistence of the CRISPRCas system and the resistance genes in 16 isolates of Enterococcus faecalis and 40 isolates of E. faecium. The negative interference between the CRISPR-Cas system and the acquisition of larger genetic structures, such as plasmids, was reported by Marraffini and Sontheimer [2008], who found within the CRISPR array of Staphylococcus epidermidis a spacer sequence corresponding to the nickase gene, present in almost all conjugative plasmids of staphylococci. The CRISPR interference was supposed to limit the HGT by preventing conjugation and plasmid transformation in staphylococci. Price et al. [2019], who studied the acquisition of pheromone-responsive plasmids in the species E. faecalis, have come to similar conclusions regarding the process of CRISPR-mediated plasmid acquisition. It was noted that the system prevents the acquisition of these plasmids in approximately $85 \%$ of the isolates in vivo (from the gastrointestinal tract of mice), but it has a much lower effect on the plasmids under different in vitro conditions (planktonic and biofilm). One study on an impressive collection of Pseudomonas aeruginosa isolates [van Belkum et al., 2015] showed that genomes that have types I-F and I-E of the CRISPR-Cas system are shorter by about $300 \mathrm{~kb}$ than those without the system. This difference, which consists of approximately 22,036 genes, was called the accessory genome and was

CRISPR-Cas: The Immunomodulatory

System in Prokaryotes found to be DNA targeted by CRISPR-Cas systems. In $P$. aeruginosa, active CRISPR-Cas systems are associated with smaller genomes and higher GC content. This suggests that CRISPR-Cas inhibits the acquisition of foreign DNA, since MGEs usually have lower GC content than their bacterial hosts. Another analysis of 300 P. aeruginosa genomes led to the conclusion that CRISPR-Cas restricts HGT, and the evolutionary mechanisms that ensure its maintenance or drive its loss are key to the ability of this pathogen to adapt to new niches and stressors [Wheatley and MacLean, 2020].

However, the relationship between CRISPR-Cas systems and HGT has not yet been elucidated. Despite the earlier lines of evidence that suggested the impairment of HGT by the CRISPR-Cas system, Gophna et al. [2015] assumed a more subtle relationship between the magnitude of HGT and the activity of CRISPR-Cas at the evolutionary scale. They tested the hypothesis that the activity of the CRISPR-Cas system would be negatively correlated with the magnitude of the HGT phenomenon. To assess the activity of the system, they considered the length of the CRISPR array: a large number of spacer and repeat sequences denoted a strong activity, but no statistically significant dependence was found between the number of recent HGT events and the activity of the CRISPRCas system. Most likely, the massive limitation of HGT would mean too high cost for the fitness of the CRISPRCas system, and it would no longer be maintained [McGinn and Marraffini, 2019]. Therefore, the events in which the CRISPR-Cas system controls the acquisition of resistance genes occur rather on a population scale and not on an evolutionary scale. On the other hand, selection for mobility of CRISPR-Cas components by MGEs contributes to the evolution of CRISPR-Cas systems and of bacteria as well.

\section{HGT of the CRISPR-Cas System}

Recent outcomes also revealed new directions with respect to the relationship between CRISPR-Cas systems and HGT. In archaea, besides spacers matching MGEs, such as plasmids or viruses, other spacers were found to match non-self chromosomal genes, providing evidence for the extensive inter-genus and inter-species HGT [Brodt et al., 2011]. Located on chromosomes, plasmids, or genomic islands, CRISPR-Cas systems as well are disseminated horizontally by conjugation or transduction [Watson et al., 2018; Varble et al., 2019]. Despite the ability of CRISPR-Cas systems to limit HGT through conjugation, transformation, and transduction, their main role in viral/bacteriophage resistance was demonstrated to promote HGT. A 
complex dynamics between CRISPR-Cas-mediated phage resistance and transduction leads to the mobilization of a chromosomal CRISPR-Cas system containing phage-targeting spacers and enhances generalized transduction [Watson et al., 2018]. Protospacers can recombine with spacers in either chromosomal or plasmid-borne CRISPR loci, leading to either the transfer of CRISPR-adjacent genes or the propagation of acquired immunity to other bacteria in the population, respectively [Varble et al., 2019]. A comparative genomic and phylogenetic analysis of CRISPR-Cas variants associated with distinct families of transposable elements generated the hypothesis that they contribute to the propagation of these MGEs by facilitating transposition into specific sites. As it was previously shown, three distinct groups of Tn7-like transposons that encode different variants of type I CRISPR-Cas systems were identified, and a new mechanism of RNA-guided transposition was described for DNA transposons [Peters et al., 2017]. More recently, CRISPR-Cas systems were detected predominantly on MGEs such as genomic islands, plasmids, and transposon-like elements. Phylogenetic analysis of Cas proteins indicated that the CRISPR-Cas systems were acquired by HGT [McDonald et al., 2019]. The new paradigm is the mechanism by which CRISPR-Cas systems associated with transposons recognize protospacers and, instead of destroying them, transpose adjacent to them [Strecker et al., 2019]. Bacterial Tn7-like transposons have coopted nuclease-deficient CRISPR-Cas systems to catalyze RNA-guided integration of MGEs into the genome. Integration of donor DNA occurs highly specifically [Klompe et al., 2019]. The precision of RNA-guided DNA insertion with CRISPR-associated transposases and the discovery of a fully programmable integrase lays the foundation for genomic manipulations that obviate the requirements for double-strand breaks and homology-directed repair [Klompe et al., 2019; Strecker et al., 2019]. Having an important role in the ecology and evolution of microbial pangenomes [Brockhurst et al., 2019], due to the variation induced by accessory genes such as MGEs, HGT is a risky evolutionary strategy. Recruitment of CRISPR-Cas systems by MGEs is a common phenomenon under which defence systems are repeatedly recruited for offence activities [Faure et al., 2019].

\section{CRISPR-Cas Regulation of Virulence Gene Expression in Bacteria}

During their infection cycle, pathogenic bacteria encounter different stress conditions and the presence of foreign DNA elements; thus, it is expected to rely on ef- ficient defence systems such as CRISPR-Cas. Virulence, as a specific stress response of pathogens, results in the expression of virulence genes modulated by CRISPR-Cas system [Louwen et al., 2014]. Most pathogens involved in nosocomial infections possess biofilm-forming abilities, which play major roles in virulence and drug resistance. An increased ability to form biofilms was observed in CRISPR-Cas-positive strains of E. faecalis [Bourgogne et al., 2008] and P. aeruginosa [Zegans et al., 2009]. CRISPRCas gene expression is induced by quorum sensing in Serratia marcescens [Patterson et al., 2016], P. aeruginosa [Høyland-Kroghsbo et al., 2017] and Clostridium difficile [Maikova et al., 2018]. In Acinetobacter baumannii, specific genes involved in biofilm formation appear almost exclusively in strains enriched in CRISPR-Cas systems [Mangas et al., 2019]. Deletion of the cas3 gene in Streptococcus mutans affects biofilm formation [Tang et al., 2019], leading to diminished growth under oxidative stress, enhanced growth under low $\mathrm{pH}$, and reduced survival under heat shock and DNA-damaging conditions [Serbanescu et al., 2015]. In $P$. aeruginosa, the cas 3 gene is involved in the upregulation of virulence factors allowing the bacteria to evade recognition by the host immune system [Li et al., 2016]. In Legionella pneumophila, the cas 2 gene is required for intracellular infection [Gunderson and Cianciotto, 2013]. Downregulation of biofilm formation ability and reduction of intracellular invasion by deletion of cas3 were also demonstrated in Salmonella enterica [Cui et al., 2020]. Cas9 gene is important for the virulence of Campylobacter jejuni [Louwen et al., 2013; Shabbir et al., 2018], Francisella novicida and Neisseria meningitidis [Sampson et al., 2013; Heidrich et al., 2019], affecting adhesion, invasion and intracellular survival. In Streptococcus pyogenes, deletion of the cas 9 gene was linked to a reduction in the abundance of virulence determinants and the expression of several virulence regulatory proteins. The mutant strain has reduced adherence to epithelial cells and other altered parameters such as growth in human whole blood ex vivo, and virulence in a murine necrotizing skin infection model, compared to the wild-type parent [Gao et al., 2019]. Although the presence of cas genes was found to correlate with the upregulation of certain virulence factors, the complex role of the CRISP-Cas systems in major pathogens remains to be discovered. A positive correlation between the virulence of $P$. aeruginosa and the presence of CRISPR-Cas systems was observed, but CRISPR-Cas activity is neither necessary nor sufficient for increased virulence. The findings of Vasquez-Rifo et al. [2019] suggest that bacterial adaptive immunity and virulence are indirectly associated 
with the effects of physiological, ecological, and evolutionary factors.

It was demonstrated that the CRISPR-Cas immune system prevented the virulence acquisition of Streptococcus pneumoniae [Bikard et al., 2012]. In E. coli, the negative correlation between the number of CRISPR units and the presence of pathogenicity traits was associated with the repeat content [García-Gutiérrez et al., 2015]. Investigating the impact of CRISPR-Cas systems in the Bacillus cereus group, they were found to be barriers to HGT, being selectively inactivated during bacterial evolution, to allow the acquisition of MGEs for adaptation to diverse environments. Introduction of a functional CRISPR-Cas system into a strain lacking the system resulted in reduced adaptability to various stresses and decreased pathogenicity [Zheng et al., 2020].

\section{Anti-CRISPR Defence}

As in any other prey-predator relationship, bacteria and bacteriophages constantly exert pressure on each other, which leads to a co-dependent evolution: bacteria find new ways to survive, and phages develop new ways to avoid or counteract bacterial defence mechanisms. The bacterial defence against bacteriophages is based both on innate immunity [Seed, 2015; Trasanidou et al., 2019] found in all bacteria, and on the acquired immunity, represented by the CRISPR-Cas system. The innate immunity has low efficiency, while the CRISPR-Cas system is a considerable hindrance to phage invasion. Therefore, phages have developed mechanisms to escape this defence by encoding proteins that inactivate the system, which have been called anti-CRISPR proteins [BondyDenomy et al., 2013; Pawluk et al., 2014; Bondy-Denomy et al., 2015].

The first genes for anti-CRISPR proteins (Acr) have been identified in phages that infect $P$. aeruginosa with the type I-F CRISPR system [Bondy-Denomy et al., 2013]. Since then, numerous studies focused on finding proteins which also inactivate other types. To date, there are known proteins capable to inactivate several CRISPR types: I-C, I-D, I-E, I-F, II-A, II-C, V-A, and VI-B. For more details regarding the protein structure, mechanism of action, and bacterial species where they have been identified, see Trasanidou et al. [2019]. Recently, it has been discovered that a bacteriophage is able to form a nuclease-like structure, where it can hide from the DNAtargeting CRISPR immunity, but remains susceptible to RNA-targeting CRISPR-Cas systems [Malone et al., 2020;

CRISPR-Cas: The Immunomodulatory

System in Prokaryotes
Mendoza et al., 2020]. One of those RNA-targeting systems is the type III CRISPR-Cas, for which a mechanism by which bacteriophages can subvert it has very recently been uncovered [Athukoralage et al., 2020]. The latest studies are focused on finding proteins that inactivate CRISPR-Cas9 systems used in genetic editing. CRISPRCas9 represents the most powerful and specific gene editing instrument currently available, even though one possible risk of this tool might be the nonspecific action of the system, which could occur over time. In these conditions, finding an off-switch for the system is as useful as it can be [Pawluk et al., 2016b; Rauch et al., 2017; Dong et al., 2017].

In addition to being located on phages, $A c r$ genes have been found in other mobile DNA structures, such as plasmids and transposons, which also carry out HGT. It was shown that these genes are located in the genome of bacterial cells holding CRISPR-Cas systems, and most likely they come from MGEs, being kept in the bacterial genome because a temporary inactivation of the system can confer an advantage on the acquisition of beneficial genes [Pawluk et al., 2016a].

\section{Biotechnological Applications}

Besides the CRISPR-Cas-based research on genetic editing and cancer therapy, which started years ago, the latest research focuses on using the system to combat pathogens, such as bacteria or viruses, and many other biotechnological applications [Carroll and Zhou, 2017]. Self-targeting by CRISPR-Cas was previously found to be lethal in bacteria. By analyzing CRISPRs from 330 organisms, Stern et al. [2010] found that self-targeting occurs in $18 \%$ of all CRISPR-bearing organisms. Therefore, the use of CRISPR-Cas systems to combat bacterial pathogens was suggested by transferring the CRISPR loci targeting chromosomal regions associated with virulence or antibiotic resistance [Bikard et al., 2012]. The RNA-programmable genome editing using the Cas9 endonuclease proposed by Jinek et al. [2012] has been widely adopted. Stern et al. [2010], observed the abundance of degraded repeats near self-targeting spacers and no conservation across species; thus, they suggested that self-targeting is a form of autoimmunity rather than a regulatory mechanism that incurs an autoimmune fitness cost and could explain the abundance of degraded CRISPR systems across prokaryotes [Stern et al., 2010]. Therefore, CRISPR-Cas systems have been successfully repurposed to target pathogenic bacteria. Removal of individual bacterial 
strains from mixed populations of $E$. coli opened new avenues for the development of "smart" antibiotics that prevent resistance and differentiate between pathogenic and beneficial microorganisms [Gomaa et al., 2014]. Such effective tools are the CRISPR-Cas3 and CRISPR-Cas9 constructs, which can be designed to target specific virulence genes and thus to attack pathogens, or to target antibiotic resistance genes and thus to fight drug resistance. This strategy is efficient in the case of chromosomal genes but not in the case of plasmid-encoded virulence and resistance, but research never stops. A series of CRISPRCas13a-based antibacterial nucleocapsids capable to kill resistant pathogens by recognizing antimicrobial resistance genes have recently been developed. CapsidCas13a constructs generated by packaging programmed CRISPR-Cas13a from Leptotrichia shahii into a bacteriophage capsid demonstrated sequence-specific killing by RNase activity. The systems were proposed as both therapeutic agents against bacterial infections and diagnostic agents to detect bacterial resistance genes [Kiga et al., 2020]. An interesting perspective of programmed CRISPR-Cas systems with discriminatory power between pathogens and commensal bacteria could be the modification of microbial communities (i.e., the human microbiome associated with different diseases).

A possible resistance could also be developed here, in time, under the selective pressure of using CRISPR as antimicrobials, due to the existence of Acr genes that we mentioned in the previous section. Development of the self-targeting gene editing technology allowed to target different positions of the virulence genes. In S. mutans, precise modification of glucosyltransferases resulted in decreased exopolysaccharide synthesis and a weakened ability to form biofilms [Gong et al., 2018]. CRISPR-Cas technology can be applied to create antimicrobials with the spectrum of activity chosen by design. RNA-guided nucleases targeting specific DNA sequences were delivered efficiently, improving survival in a Galleria mellonella infection model [Citorik et al., 2014]. The phage-based approach was also used to deliver a CRISPR-Cas9 system targeting the chromosomal kanamycin resistance gene in Staphylococcus aureus. As a result, a strong growth inhibition of resistant bacteria due to chromosome cleavage and subsequent cell death were obtained. In an in vivo mouse skin infection model, the treatment also led to a significant reduction of antibiotic-resistant bacteria [Bikard et al., 2014].

A new CRISPR-Cas9-mediated genome editing method and a base editing system were recently developed, enabling the cytidine base editor to inactivate a target gene by generating a premature stop codon. The highly efficient genetic manipulation in Pseudomonas species is estimated to accelerate a wide variety of investigations of bacterial physiology, drug target exploration, and metabolic engineering [Chen et al., 2018].

In addition, CRISPR interference (CRISPRi), a genetic perturbation technique, allows the sequence-specific repression of gene expression in prokaryotic and eukaryotic cells [Qi et al., 2013]. The CRISPRi mechanism was applied in E. coli for inhibiting bacterial biofilm by silencing the luxS gene encoding a synthase involved in the initial stage of biofilm formation [Zuberi et al., 2017]. The identification of rules for specific targeting of transcriptional repressors (CRISPRi) and activators (CRISPRa) to endogenous genes via endonuclease-deficient Cas9 laid new foundations to enable modulation of gene expression [Gilbert et al., 2014]. CRISPR-Cas systems have been successfully repurposed to target virulence factors and antibiotic resistance genes in bacteria and constitute an appealing option for programmable and sequence-specific antimicrobials [Bikard and Barrangou, 2017]. Genome editing became the major outcome of self-targeting using CRISPR-based technologies in eukaryotes. Targeted gene regulation is now used to influence the expression of genes in human cells for cell and gene therapy, genetic reprogramming, and regenerative medicine.

CRISPR-Cas system has been proposed for treating, besides bacterial infections, also viral infections. CRISPRCas9 has been engineered for combating human viruses, such as Epstein-Barr virus, human papillomavirus, and hepatitis B virus, all of this being DNA viruses. Since Cas9 cannot interact directly with RNA, CRISPR-Cas9 is not efficient for curing retroviral infections [Li et al., 2020]. Considering the current situation regarding the COVID-19 pandemic, caused by the retrovirus SARS-CoV-2, the CRISPR research is focusing on engineering a system able to target RNA, which can be used as a treatment. Recent studies showed that Cas13 proteins, Cas13a [Li et al., 2020] and Cas13d [Nguyen et al., 2020], are able to target RNA. Nguyen et al. were able to design efficient guide RNAs, which target the coding regions of the novel coronavirus. This guide RNAs are also safe since they do not affect the human transcriptome. A study available online as a preprint, while still under review, claimed that they managed to engineer a system able to target over $90 \%$ of all coronaviruses, which could be quickly used in other rising strains that could cause a pandemic [Abbott et al., 2020]. Another promising application for CRISPR-Cas systems in the context of the ongoing pandemic, is for the surveillance of the disease. The most recent research papers proposed 
some diagnostic tools for SARS-CoV-2, alongside other RNA viruses [Metsky et al., 2020; Ding et al., 2020; Curti et al., 2020]. This tools are based on the SHERLOCK method (specific high-sensitivity enzymatic reporter unlocking) developed by Kellner et al. in 2019. This method is based on the capacity of Cas 12 and Cas 13 proteins to cleave unspecific RNA in their proximity, if the associated crRNA finds its target, which can lead to a color or florescent signal emission, when the DNA or RNA target is present. The specificity of the test depends on the crRNA design. Several crRNAs have been designed to target specific sequences in the SARS-CoV-2 [Metsky et al., 2020; Ding et al., 2020; Curti et al., 2020], other human coronaviruses [Metsky et al., 2020], and HIV [Ding et al., 2020]. These diagnostic tools seem to be rapid, highly specific, comparable in sensitivity to the qPCR method, and possibly portable.

\section{Conclusion}

Despite the numerous studies, the CRISPR-Cas system is still of great interest, several aspects are far less clear than others, such as the origin of the effector modules and the mechanism of spacer integration/deletion. Although the evolution of adaptation and effector modules seem to be partially independent, and the origin of the adaptation module in casposons is accepted, little is known about the effector module. The classification of CRISPR-Cas systems in 2 classes is based mostly on the organization of the effector module. The class 1 systems have multisubunit effectors with origin in the nucleases encoded by different MGEs. The class 2 systems, in which the effector consists of a single, large protein, have an uncertain ori- gin. The contribution of MGEs to the recruitment of minimalist variants of CRISPR-Cas systems remains to be elucidated. Moreover, phylogenomic studies revealed a continuous diversification of the CRISPR-Cas systems and their adaptation modules as well, which needs more attention as well.

The role of the CRISPR-Cas system in the modulation of genotypes, physiology, and ecology of prokaryotes, the implication of HGT in the acquisition of CRISPR-Cas modules, and the role of the CRISPR-Cas system in the limitation of HGT, to allow the acquisition of advantageous genes are topics of great interest, as well as the development of new applications in the field of diagnostic and treatment of infectious diseases.

\section{Conflict of Interest Statement}

The authors have no conflicts of interest to declare.

\section{Funding Sources}

This work was supported by Babeş-Bolyai University, Cluj-Napoca, Romania. This research did not receive any specific grants from funding agencies in the public, commercial, or not-for-profit sectors.

\section{Author Contributions}

Anca Butiuc-Keul: conceptualization, original draft preparation, and supervision. Anca Farkas: writing-reviewing and editing. Rahela Carpa: writing-reviewing. Dumitrana Iordache: writingreviewing and editing.

\section{References}

Abbott TR, Dhamdhere G, Liu Y, Lin X, Goudy L, Zeng L, et al. Development of CRISPR as an Antiviral Strategy to Combat SARS-CoV-2 and Influenza. Cell. 2020;181:865-e12.

Amitai G, Sorek R. CRISPR-Cas adaptation: Insights into the mechanism of action. Nat Rev Microbiol. 2016;14:67-76.

Arslan Z, Hermanns V, Wurm R, Wagner R, Pul $\ddot{U}$. Detection and characterization of spacer integration intermediates in type I-E CRISPR-Cas system. Nucleic Acids Res. 2014;42: 7884-93.

Athukoralage JS, McMahon SA, Zhang C, Grüschow S, Graham S, Krupovic M, et al. An anti-CRISPR viral ring nuclease subverts type III CRISPR immunity. Nature. 2020;577:5725.
Babu M, Beloglazova N, Flick R, Graham C, Skarina T, Nocek B, et al. A dual function of the CRISPR-Cas system in bacterial antivirus immunity and DNA repair. Mol Microbiol. 2011;79:484-502.

Barrangou R, Fremaux C, Deveau H, Richards M, Boyaval P, Moineau S, et al. CRISPR provides acquired resistance against viruses in prokaryotes. Science. 2007;315:1709-12.

Barrangou R, Marraffini LA. CRISPR-cas systems: Prokaryotes upgrade to adaptive immunity. Mol Cell. 2014;54:234-44.

Barrangou R, Horvath P. A decade of discovery: CRISPR functions and applications. Nat Microbiol. 2017;2:17092-9.
Béguin $\mathrm{PB}^{\prime}$, Charpin N, Koonin EV, Forterre P, Krupovic M. Casposon integration shows strong target site preference and recapitulates protospacer integration by CRISPRCas systems. Nucleic Acids Res. 2016;44: 10367-76.

van Belkum A, Soriaga LB, LaFave MC, Akella S, Veyrieras JB, Barbu EM, et al. Phylogenetic Distribution of CRISPR-Cas Systems in Antibiotic-Resistant Pseudomonas aeruginosa MBio. 2015;6:e1796.

Bhaya D, Davison M, Barrangou R. CRISPR-Cas systems in Bacteria and Archaea: Versatile small RNAs for adaptive defense and regulation. Annu Rev Genet. 2011;45:273-97. 
Bikard D, Hatoum-Aslan A, Mucida D, Marraffini LA. CRISPR interference can prevent natural transformation and virulence acquisition during in vivo bacterial infection. Cell Host Microbe. 2012;12:177-86.

Bikard D, Euler CW, Jiang W, Nussenzweig PM, Goldberg GW, Duportet X, et al. Exploiting CRISPR-cas nucleases to produce sequencespecific antimicrobials. Nat Biotechnol. 2014; 32:1146-50.

Bikard D, Barrangou R. Using CRISPR-Cas systems as antimicrobials. Curr Opin Microbiol. 2017;37:155-60.

Bondy-Denomy J, Pawluk A, Maxwell KL, Davidson AR. Bacteriophage genes that inactivate the CRISPR/Cas bacterial immune system. Nature. 2013;493:429-32.

Bondy-Denomy J, Garcia B, Strum S, Du M, Rollins MF, Hidalgo-Reyes Y, et al. Multiple mechanisms for CRISPR-Cas inhibition by antiCRISPR proteins. Nature. 2015;526:136-9.

Bourgogne A, Garsin DA, Qin X, Singh KV, Sillanpaa J, Yerrapragada S, et al. Large scale variation in Enterococcus faecalis illustrated by the genome analysis of strain OG1RF. Genome Biol. 2008;9:R110

Brockhurst MA, Harrison E, Hall JPJ, Richards T, McNally A, MacLean C. The ecology and evolution of pangenomes. Curr Biol. 2019;29(20): R1094-103.

Brodt A, Lurie-Weinberger MN, Gophna U. CRISPR loci reveal networks of gene exchange in archaea. Biol Direct. 2011;6:65.

Brouns SJ, Jore MM, Lundgren M, Westra ER, Slijkhuis RJ, Snijders AP, et al. Small CRISPR RNAs guide antiviral defense in prokaryotes. Science. 2008;321(80)960-4.

Carroll M, Zhou X. Panacea in progress: CRISPR and the future of its biological research introduction. Microbiol Res. 2017;201:63-74.

Charpentier E, Richter H, Van Der Oost J, White MF. Biogenesis pathways of RNA guides in archaeal and bacterial CRISPR-Cas adaptive immunity. FEMS Microbiol Rev. 2015;39: $428-41$.

Chen W, Zhang Y, Zhang Y, Pi Y, Gu T, Song L, et al. CRISPR/Cas9-based genome editing in Pseudomonas aeruginosa and cytidine deaminase-mediated base editing in Pseudomonas species. iScience. 2018;6:222-31.

Chylinski K, Le Rhun A, Charpentier E. The tracrRNA and Cas9 families of type II CRISPR-Cas immunity systems. RNA Biol. 2013; 10:726-37.

Citorik RJ, Mimee M, Lu TK. Sequence-specific antimicrobials using efficiently delivered RNA-guided nucleases. Nat Biotechnol. 2014; 32:1141-5.

Cox DBT, Gootenberg JS, Abudayyeh OO, Franklin B, Kellner MJ, Joung J, et al. RNA editing with CRISPR-Cas13. Science. 2017;358: 1019-27.

Cui L, Wang X, Huang D, Zhao Y, Feng J, Lu Q, et al. CRISPR-cas3 of Salmonella upregulates bacterial biofilm Formation and virulence to host cells by targeting quorum-sensing systems. Pathogens. 2020;9:53.
Curti L, Pereyra-Bonnet F, Gimenez C. An ultrasensitive, rapid, and portable coronavirus SARS-CoV-2 sequence detection method based on CRISPR-Cas12. bioRxiv. 2020;2020. 02.29.971127.

Dai W, Xu X, Wang D, Wu J, Wang J. Cancer therapy with a CRISPR-assisted telomeraseactivating gene expression system. Oncogene. 2019;38:4110-24.

Díez-Villaseñor C, Guzmán NM, Almendros C, García-Martínez J, Mojica FJ. CRISPR-spacer integration reporter plasmids reveal distinct genuine acquisition specificities among CRISPR-Cas I-E variants of Escherichia coli. RNA Biol. 2013;10:792-802.

Ding X, Yin K, Li Z, Liu C. All-in-one dual CRISPR-Cas12a (AIOD-CRISPR) assay: A case for rapid, ultrasensitive and visual detection of novel coronavirus SARS-CoV-2 and HIV virus. bioRxiv. 2020;2020. .03.19.998724.

Dong D, Guo M, Wang S, Zhu Y, Wang S, Xiong $Z$, et al. Structural basis of CRISPR-SpyCas9 inhibition by an anti-CRISPR protein. Nature. 2017;546:436-9.

Doron S, Melamed S, Ofir G, Leavitt A, Lopatina A, Keren M, et al. Systematic discovery of antiphage defense systems in the microbial pangenome. Science. 2018:359.

Doudna JA, Charpentier E. Genome editing. The new frontier of genome engineering with CRISPR-Cas9. Science. 2014;346:1258096.

Faure G, Shmakov SA, Yan WX, Cheng DR, Scott DA, Peters JE, et al. CRISPR-Cas in mobile genetic elements: counter-defence and beyond. Nat Rev Microbiol. 2019;17:513-25.

Fineran PC, Charpentier E. Memory of viral infections by CRISPR-Cas adaptive immune systems: Acquisition of new information. Virology. 2012;434:202-9.

Gao NJ, Al-Bassam MM, Poudel S, Wozniak JM, Gonzalez DJ, Olson J, et al. Functional and proteomic analysis of Streptococcus pyogenes virulence upon loss of its native Cas9 nuclease. Front Microbiol. 2019;10:1967.

García-Gutiérrez E, Almendros C, Mojica FJ, Guzmán NM, García-Martínez J. CRISPR content correlates with the pathogenic potential of Escherichia coli. PLoS One. 2015;10: e0131935.

García-Martínez J, Maldonado RD, Guzmán NM, Mojica FJM. The CRISPR conundrum: Evolve and maybe die, or survive and risk stagnation. Microb Cell. 2018;5:262-8.

Gilbert LA, Horlbeck MA, Adamson B, Villalta JE, Chen Y, Whitehead EH, et al. Genomescale CRISPR-mediated control of gene repression and activation. Cell. 2014;159:64761.

Gleditzsch D, Pausch P, Müller-Esparza H, Özcan A, Guo X, Bange G, et al. PAM identification by CRISPR-Cas effector complexes: diversified mechanisms and structures. RNA Biol. 2019;16:504-17.

Gomaa AA, Klumpe HE, Luo ML, Selle K, Barrangou R, Beisel CL. Programmable removal of bacterial strains by use of genome-targeting CRISPR-Cas systems. MBio. 2014;5:e00928.
Gong T, Tang B, Zhou X, Zeng J, Lu M, Guo X, et al. Genome editing in Streptococcus mutans through self-targeting CRISPR arrays. Mol Oral Microbiol. 2018;33:440-9.

Gophna U, Kristensen DM, Wolf YI, Popa O, Drevet C, Koonin EV. No evidence of inhibition of horizontal gene transfer by CRISPRCas on evolutionary timescales. ISME J. 2015; 9:2021-7.

Grissa I, Vergnaud G, Pourcel C. CRISPRFinder: a web tool to identify clustered regularly interspaced short palindromic repeats. Nucleic Acids Res. 2007;35:W52.

Gunderson FF, Cianciotto NP. The CRISPR-associated gene cas 2 of Legionella pneumophila is required for intracellular infection of amoebae. MBio. 2013;4:e00074.

Haft DH, Selengut J, Mongodin EF, Nelson KE. A Guild of 45 CRISPR-Associated (Cas) Protein Families and Multiple CRISPR/Cas Subtypes Exist in Prokaryotic Genomes. PLoS Comput Biol. 2005; 1:e60.

Hampton HG, Watson BNJ, Fineran PC. The arms race between bacteria and their phage foes. Nature. 2020;577:327-36.

Han P, Niestemski LR, Barrick JE, Deem MW. Physical model of the immune response of bacteria against bacteriophage through the adaptive CRISPR-Cas immune system. Phys Biol. 2013;10:025004.

Heidrich N, Hagmann A, Bauriedl S, Vogel J, Schoen C. The CRISPR/Cas system in Neisseria meningitidis affects bacterial adhesion to human nasopharyngeal epithelial cells. RNA Biol. 2019;16:390-6.

Heler R, Samai P, Modell JW, Weiner C, Goldberg GW, Bikard D, et al. Cas9 specifies functional viral targets during CRISPR-Cas adaptation. Nature. 2015;519:199-202.

Hille F, Charpentier E. CRISPR-cas: Biology, mechanisms and relevance. Philos Trans R Soc Lond B Biol Sci. 2016;371.

Hille F, Richter H, Wong SP, Bratovič M, Ressel S, Charpentier E. The Biology of CRISPR-Cas: Backward and Forward. Cell. 2018;172:1239_ 59.

Horvath P, Romero DA, Coûté-Monvoisin AC, Richards M, Deveau H, Moineau S, et al. Diversity, activity, and evolution of CRISPR loci in Streptococcus thermophilus. J Bacteriol. 2008; 190:1401-12.

Horvath P, Barrangou R. CRISPR/Cas, the immune system of Bacteria and Archaea. Science. 2010;327:167-70.

Høyland-Kroghsbo NM, Paczkowski J, Mukherjee S, Broniewski J, Westra E, Bondy-Denomy $\mathrm{J}$, et al. Quorum sensing controls the Pseudomonas aeruginosa CRISPR-Cas adaptive immune system. Proc Natl Acad Sci USA. 2017; 114:131-5.

Hsu PD, Lander ES, Zhang F. Development and applications of CRISPR-Cas9 for genome engineering. Cell. 2014;157:1262-78.

Hudaiberdiev S, Shmakov S, Wolf YI, Terns MP, Makarova KS, Koonin EV. Phylogenomics of Cas4 family nucleases. BMC Evol Biol. 2017; $17: 232$. 
Hullahalli K, Rodrigues M, Schmidt BD, Li X, Bhardwaj P, Palmer KL. Comparative analysis of the orphan CRISPR2 locus in 242 Enterococcus faecalis Strains. PLoS One. 2015; 10(9):e0138890.

Huo Y, Nam KH, Ding F, Lee H, Wu L, Xiao Y, et al. Structures of CRISPR Cas 3 offer mechanistic insights into Cascade-activated DNA unwinding and degradation. Nat Struct Mol Biol. 2014;21:771-7.

Ishino Y, Shinagawa H, Makino K, Amemura M, Nakata A. Nucleotide sequence of the iap gene, responsible for alkaline phosphatase isozyme conversion in Escherichia coli, and identification of the gene product. J Bacteriol. 1987;169:5429-33.

Jackson SA, McKenzie RE, Fagerlund RD, Kieper SN, Fineran PC, Brouns SJ. CRISPR-Cas: Adapting to change. Science. 2017;356(80): 356.

Jansen R, Embden JD, Gaastra W, Schouls LM Identification of genes that are associated with DNA repeats in prokaryotes. Mol Microbiol. 2002;43:1565-75.

Jinek M, Chylinski K, Fonfara I, Hauer M, Doudna JA, Charpentier E. A programmable dualRNA-guided DNA endonuclease in adaptive bacterial immunity. Science. 2012;(80):337: $816-21$.

Jorth P, Whiteley M. An evolutionary link between natural transformation and CRISPR adaptive immunity. MBio. 2012;3:5.

Kellner MJ, Koob JG, Gootenberg JS, Abudayyeh OO, Zhang F. SHERLOCK: nucleic acid detection with CRISPR nucleases. Nat Protoc. 2019;14:2986-3012.

Kiga K, Tan XE, Ibarra-Chávez R, Watanabe S, Aiba Y, Sato'o Y, et al. Development of CRISPR-Cas13a-based antimicrobials capable of sequence-specific killing of target bacteria. Nat Commun. 2020;11:2934.

Klompe SE, Vo PLH, Halpin-Healy TS, Sternberg SH. Transposon-encoded CRISPR-Cas systems direct RNA-guided DNA integration. Nature. 2019;571:219-25.

Kolli N, Lu M, Maiti P, Rossignol J, Dunbar GL. Application of the gene editing tool, CRISPRCas9, for treating neurodegenerative diseases. Neurochem Int. 2018;112:187-96.

Komor AC, Badran AH, Liu DR. CRISPR-based technologies for the manipulation of eukaryotic genomes. Cell. 2017;169:559-36.

Koonin EV, Makarova KS, CRISPR-Cas . CRISPR-Cas: evolution of an RNA-based adaptive immunity system in prokaryotes. RNA Biol. 2013;10:679-86.

Koonin EV, Krupovic M. Evolution of adaptive immunity from transposable elements combined with innate immune systems. Nat Rev Genet. 2015;16:184-92.

Koonin EV, Makarova KS, Zhang F. Diversity, classification and evolution of CRISPR-Cas systems. Curr Opin Microbiol. 2017;37:6778.

Koonin EV. Hunting for treasure chests in microbial defense islands. Mol Cell. 2018;70(5): 761-2.
Koonin EV, Makarova KS. Origins and evolution of CRISPR-Cas systems. Philos Trans R Soc Lond B Biol Sci. 2019;374:20180087.

Krupovic M, Makarova KS, Forterre P, Prangishvili D, Koonin EV. Casposons: A new superfamily of self-synthesizing DNA transposons at the origin of prokaryotic CRISPR-Cas immunity. BMC Biol. 2014;12:36.

Krupovic M, Koonin EV. Self-synthesizing transposons: Unexpected key players in the evolution of viruses and defense systems. Curr Opin Microbiol. 2016;31:25-33.

Krupovic M, Béguin P, Koonin EV. Casposons: mobile genetic elements that gave rise to the CRISPR-Cas adaptation machinery. Curr Opin Microbiol. 2017;38:36-43.

Kunin V, Sorek R, Hugenholtz P. Evolutionary conservation of sequence and secondary structures in CRISPR repeats. Genome Biol. 2007;8:R61.

Li H, Wang S, Dong X, Li Q, Li M, Li J, et al. CRISPR-Cas13a cleavage of dengue virus NS3 gene efficiently inhibits viral replication. Mol Ther Nucleic Acids. 2020;19:1460-9.

Li R, Fang L, Tan S, Yu M, Li X, He S, et al. Type I CRISPR-Cas targets endogenous genes and regulates virulence to evade mammalian host immunity. Cell Res. 2016;26:1273-87.

Liu T, Liu Z, Ye Q, Pan S, Wang X, Li Y, et al. Coupling transcriptional activation of CRISPR-Cas system and DNA repair genes by Csa3a in Sulfolobus islandicus. Nucleic Acids Res. 2017;45:8978-92.

Liu T, Pan S, Li Y, Peng N, She Q. Type III CRISPR-Cas system: Introduction and its application for genetic manipulations. Curr Issues Mol Biol. 2018;26:1-14.

Louwen R, Horst-Kreft D, De Boer AG, Van Der Graaf L, De Knegt G, Hamersma M, et al. A novel link between Campylobacter jejuni bacteriophage defence, virulence and GuillainBarré syndrome. Eur J Clin Microbiol Infect Dis. 2013;32:207-26.

Louwen R, Staals RH, Endtz HP, van Baarlen P, van der Oost J. The role of CRISPR-Cas Systems in virulence of pathogenic bacteria. Microbiol Mol Biol Rev. 2014;78:74-88.

Maier LK, Lange SJ, Stoll B, Haas KA, Fischer S, Fischer E, et al. Essential requirements for the detection and degradation of invaders by the Haloferax volcanii CRISPR/Cas system I-B. RNA Biol. 2013;10(5):865-74.

Maikova A, Severinov K, Soutourina O. New insights into functions and possible applications of Clostridium difficile CRISPR-Cas system. Front Microbiol. 2018;9:1740.

Makarova KS, Aravind L, Grishin NV, Rogozin IB, Koonin EV. A DNA repair system specific for thermophilic archaea and bacteria predicted by genomic context analysis. Nucleic Acids Res. 2002;30:482-96.

Makarova KS, Grishin NV, Shabalina SA, Wolf YI, Koonin EV. A putative RNA-interference-based immune system in prokaryotes: Computational analysis of the predicted enzymatic machinery, functional analogies with eukaryotic RNAi, and hypothetical mechanisms of action. Biol Direct. 2006;1:7.
Makarova KS, Haft DH, Barrangou R, Brouns SJ, Charpentier E, Horvath P, et al. Evolution and classification of the CRISPR-Cas systems. Nat Rev Microbiol. 2011;9:467-77.

Makarova KS, Wolf YI, Koonin EV. The basic building blocks and evolution of CRISPR-Cas systems; in Biochemical Society Transactions. London: Portland Press; 2013. p. 1392-400

Makarova KS, Wolf YI, Alkhnbashi OS, Costa F, Shah SA, Saunders SJ, et al. An updated evolutionary classification of CRISPR-Cas systems. Nat Rev Microbiol. 2015;13:722-36.

Malone LM, Warring SL, Jackson SA, Warnecke C, Gardner PP, Gumy LF, et al. A jumbo phage that forms a nucleus-like structure evades CRISPR-Cas DNA targeting but is vulnerable to type III RNA-based immunity. Nat Microbiol. 2020;5:48-55.

Mandin P, Repoila F, Vergassola M, Geissmann $\mathrm{T}$, Cossart P. Identification of new noncoding RNAs in Listeria monocytogenes and prediction of mRNA targets. Nucleic Acids Res. 2007;35(3):962-74

Mangas EL, Rubio A, Álvarez-Marín R, LabradorHerrera G, Pachón J, Pachón-Ibáñez ME, et al. Pangenome of Acinetobacter baumannii uncovers two groups of genomes, one of them with genes involved in CRISPR/Cas defence systems associated with the absence of plasmids and exclusive genes for biofilm formation. Microb Genom. 2019;5.

Maniv I, Jiang W, Bikard D, Marraffini LA. Impact of different target sequences on type III CRISPR-Cas immunity. J Bacteriol. 2016;198: 941-50.

Marraffini LA, Sontheimer EJ. CRISPR interference limits horizontal gene transfer in staphylococci by targeting DNA. Science. 2008;322: 1843-5.

McDonald ND, Regmi A, Morreale DP, Borowski JD, Boyd EF. CRISPR-Cas systems are present predominantly on mobile genetic elements in Vibrio species. BMC Genomics. 2019;20:105.

McGinn J, Marraffini LA. Molecular mechanisms of CRISPR-Cas spacer acquisition. Nat Rev Microbiol. 2019;17:7-12.

Mendoza SD, Nieweglowska ES, Govindarajan S, Leon LM, Berry JD, Tiwari A, et al. A bacteriophage nucleus-like compartment shields DNA from CRISPR nucleases. Nature. 2020; 577:244-8

Metsky HC, Freije CA, Kosoko-Thoroddsen T-SF, Sabeti PC, Myhrvold C. CRISPR-based surveillance for COVID-19 using genomically-comprehensive machine learning design. bioRxiv. 2020;2020. 02.26.967026.

Mitchell JS, Glowacki J, Grandchamp AE, Manning RS, Maddocks JH. Sequence-dependent persistence lengths of DNA. J Chem Theory Comput. 2017;13:1539-55.

Mohanraju P, Makarova KS, Zetsche B, Zhang F, Koonin EV, Van Der Oost J. Diverse evolutionary roots and mechanistic variations of the CRISPR-Cas systems. Science. 2016; 353(80):aad5147
CRISPR-Cas: The Immunomodulatory System in Prokaryotes
Microb Physiol 2022;32:2-17

DOI: $10.1159 / 000516643$ 
Mojica FJ, Díez-Villaseñor C, García-Martínez J, Soria E. Intervening sequences of regularly spaced prokaryotic repeats derive from foreign genetic elements. J Mol Evol. 2005;60:174-82.

Mojica FJ, Díez-Villaseñor C, García-Martínez J, Almendros C. Short motif sequences determine the targets of the prokaryotic CRISPR defence system. Microbiology (Reading). 2009; 155:733-40.

Nam KH, Ding F, Haitjema C, Huang Q, DeLisa $\mathrm{MP}, \mathrm{Ke}$ A. Double-stranded endonuclease activity in Bacillus halodurans clustered regularly interspaced short palindromic repeats (CRISPR)-associated Cas2 protein. J Biol Chem. 2012;287:35943-52.

Nguyen TM, Zhang Y, Pandolfi PP. Virus against virus: a potential treatment for 2019-nCov (SARS-CoV-2) and other RNA viruses. Cell Res. 2020;30:189-90.

Nishimasu H, Nureki O. Structures and mechanisms of CRISPR RNA-guided effector nucleases. Curr Opin Struct Biol. 2017;43:68-78.

Palmer KL, Gilmore MS. Multidrug-resistant enterococci lack CRISPR-cas. MBio. 2010;1.

Patterson AG, Jackson SA, Taylor C, Evans GB, Salmond GPC, Przybilski R, et al. Quorum sensing controls adaptive immunity through the regulation of multiple CRISPR-Cas systems. Mol Cell. 2016;64:1102-8.

Pawluk A, Bondy-Denomy J, Cheung VH, Maxwell KL, Davidson AR. A new group of phage anti-CRISPR genes inhibits the type I-E CRISPR-Cas system of Pseudomonas aeruginosa. MBio. 2014;5:e00896.

Pawluk A, Amrani N, Zhang Y, Garcia B, Hidalgo-Reyes Y, Lee J, et al. Naturally occurring off-switches for CRISPR-Cas9. Cell. 2016a; 167:1829-e9.

Pawluk A, Staals RH, Taylor C, Watson BN, Saha $S$, Fineran PC, et al. Inactivation of CRISPRCas systems by anti-CRISPR proteins in diverse bacterial species. Nat Microbiol. 2016b; 1:16085-6.

Peters JE, Makarova KS, Shmakov S, Koonin EV. Recruitment of CRISPR-Cas systems by Tn7like transposons. Proc Natl Acad Sci U S A. 2017;114:E7358-66.

Pourcel C, Salvignol G, Vergnaud G. CRISPR elements in Yersinia pestis acquire new repeats by preferential uptake of bacteriophage DNA, and provide additional tools for evolutionary studies. Microbiology (Reading). 2005; 151: 653-63.

Price VJ, McBride SW, Hullahalli K, Chatterjee A, Duerkop BA, Palmer KL. Enterococcus faecalis CRISPR-Cas is a robust barrier to conjugative antibiotic resistance dissemination in the murine intestine. mSphere. 2019;4.

Qi LS, Larson MH, Gilbert LA, Doudna JA, Weissman JS, Arkin AP, et al. Repurposing CRISPR as an RNA-guided platform for sequence-specific control of gene expression. Cell. 2013;152:1173-83.

Rath D, Amlinger L, Rath A, Lundgren M. The CRISPR-Cas immune system: Biology, mechanisms and applications. Biochimie. 2015; 117:119-28.
Rauch BJ, Silvis MR, Hultquist JF, Waters CS, McGregor MJ, Krogan NJ, et al. Inhibition of CRISPR-Cas9 with bacteriophage proteins. Cell. 2017;168:150-e10.

Richter C, Chang JT, Fineran PC. Function and regulation of Clustered Regularly Interspaced Short Palindromic Repeats (CRISPR) / CRISPR associated (Cas) systems. Viruses. 2012;4: 2291-311.

Rollie C, Schneider S, Brinkmann AS, Bolt EL, White MF. Intrinsic sequence specificity of the Cas1 integrase directs new spacer acquisition. Elife. 2015; 4.

Rouillon C, Zhou M, Zhang J, Politis A, BeilstenEdmands V, Cannone G, et al. Structure of the CRISPR interference complex CSM reveals key similarities with cascade. Mol Cell. 2013; 52:124-34.

Samai P, Pyenson N, Jiang W, Goldberg GW, Hatoum-Aslan A, Marraffini LA. Co-transcriptional DNA and RNA cleavage during type III CRISPR-Cas immunity. Cell. 2015; 161:1164-74.

Samanta MK, Dey A, Gayen S. CRISPR/Cas9: an advanced tool for editing plant genomes. Transgenic Res. 2016;25:561-73.

Sampson TR, Saroj SD, Llewellyn AC, Tzeng YL, Weiss DS. A CRISPR/Cas system mediates bacterial innate immune evasion and virulence. Nature. 2013;497:254-7.

Schaeffer SM, Nakata PA. CRISPR/Cas9-mediated genome editing and gene replacement in plants: Transitioning from lab to field. Plant Sci. 2015;240:130-42.

Seed KD. Battling phages: How bacteria defend against viral attack. PLoS Pathog. 2015;11(6): e1004847.

Serbanescu MA, Cordova M, Krastel K, Flick R, Beloglazova N, Latos A, et al. Role of the Streptococcus mutans CRISPR-Cas systems in immunity and cell physiology. J Bacteriol. 2015; 197:749-61.

Shabbir MA, Hao H, Shabbir MZ, Wu Q, Sattar A, Yuan Z. Bacteria vs. bacteriophages: parallel evolution of immune arsenals. Front Microbiol. 2016;7:1292.

Shabbir MAB, Tang Y, Xu Z, Lin M, Cheng G, Dai $\mathrm{M}$, et al. The involvement of the Cas9 gene in virulence of Campylobacter jejuni. Front Cell Infect Microbiol. 2018;8:285.

Shah SA, Erdmann S, Mojica FJ, Garrett RA. Protospacer recognition motifs: Mixed identities and functional diversity. RNA Biol. 2013;10:891-9.

Shmakov SA, Sitnik V, Makarova KS, Wolf YI, Severinov KV, Koonin EV. The CRISPR spacer space is dominated by sequences from species-specific mobilomes. MBio. 2017;8.

Silas S, Mohr G, Sidote DJ, Markham LM, Sanchez-Amat A, Bhaya D, et al. Direct CRISPR spacer acquisition from RNA by a natural reverse transcriptase-Cas1 fusion protein. Science. 2016:351.

Silas S, Makarova KS, Shmakov S, Páez-Espino D, Mohr G, Liu Y, et al. On the Origin of Reverse Transcriptase-Using CRISPR-Cas Systems and Their Hyperdiverse, Enigmatic Spacer Repertoires. MBio. 2017;8.
Sinkunas T, Gasiunas G, Fremaux C, Barrangou $\mathrm{R}$, Horvath P, Siksnys V. Cas3 is a singlestranded DNA nuclease and ATP-dependent helicase in the CRISPR/Cas immune system. EMBO J. 2011;30:1335-42.

Soppe JA, Lebbink RJ. Antiviral goes viral: Harnessing CRISPR/Cas9 to combat viruses in humans. Trends Microbiol. 2017;25:833-50.

Sorek R, Lawrence CM, Wiedenheft B. CRISPRmediated adaptive immune systems in Bacteria and Archaea. Annu Rev Biochem. 2013;82: 237-66.

Staals RHJ, Agari Y, Maki-Yonekura S, Zhu Y, Taylor DW, van Duijn E, et al. Structure and activity of the RNA-targeting type III-B CRISPR-Cas complex of Thermus thermophilus. Mol Cell. 2013;52:135-45.

Staals RH, Zhu Y, Taylor DW, Kornfeld JE, Sharma K, Barendregt A, et al. RNA targeting by the type III-A CRISPR-Cas Csm complex of Thermus thermophilus. Mol Cell. 2014;56: 518-30.

Stern A, Keren L, Wurtzel O, Amitai G, Sorek R. Self-targeting by CRISPR: Gene regulation or autoimmunity? Trends Genet. 2010;26:33540.

Strecker J, Ladha A, Gardner Z, Schmid-Burgk JL, Makarova KS, Koonin EV, et al. RNA-guided DNA insertion with CRISPR-associated transposases. Science. 2019;365:48-53.

Takeuchi N, Wolf YI, Makarova KS, Koonin EV. Nature and intensity of selection pressure on CRISPR-associated genes. J Bacteriol. 2012; 194:1216-25.

Tang B, Gong T, Zhou X, Lu M, Zeng J, Peng X, et al. Deletion of cas3 gene in Streptococcus mutans affects biofilm formation and increases fluoride sensitivity. Arch Oral Biol. 2019; 99:190-7.

Trasanidou D, Gerós AS, Mohanraju P, Nieuwenweg AC, Nobrega FL, Staals RHJ. Keeping CRISPR in check: diverse mechanisms of phage-encoded anti-CRISPRs. FEMS Microbiol Lett. 2019;366.

Varble A, Meaden S, Barrangou R, Westra ER, Marraffini LA. Recombination between phages and CRISPR-cas loci facilitates horizontal gene transfer in staphylococci. Nat Microbiol. 2019;4:956-63.

Vasquez-Rifo A, Veksler-Lublinsky I, Cheng Z, Ausubel FM, Ambros V. The Pseudomonas aeruginosa accessory genome elements influence virulence towards Caenorhabditis elegans. Genome Biol. 2019;20:270.

Wang J, Li J, Zhao H, Sheng G, Wang M, Yin M, et al. Structural and mechanistic basis of PAM-dependent spacer acquisition in CRISPR-Cas systems. Cell. 2015;163:840-53.

Watson BNJ, Staals RHJ, Fineran PC. CRISPRcas-mediated phage resistance enhances horizontal gene transfer by transduction. MBio. 2018;9(1).

Wei Y, Terns RM, Terns MP. Cas9 function and host genome sampling in Type II - A CRISPR-Cas adaptation. Genes Dev. 2015;29:35661.

Butiuc-Keul/Farkas/Carpa/Iordache 
Wheatley RM, MacLean RC. CRISPR-Cas systems restrict horizontal gene transfer in Pseudomonas aeruginosa. ISME J. 2020.

Wright AV, Nuñez JK, Doudna JA. Biology and applications of CRISPR systems: harnessing nature's toolbox for genome engineering. Cell. 2016;164:29-44.

Wu WY, Lebbink JHG, Kanaar R, Geijsen N, Van Der Oost J. Genome editing by natural and engineered CRISPR-associated nucleases. Nat Chem Biol. 2018;14:642-51.

Yutin N, Bäckström D, Ettema TJG, Krupovic M, Koonin EV. Vast diversity of prokaryotic virus genomes encoding double jelly-roll major capsid proteins uncovered by genomic and metagenomic sequence analysis. Virol J. 2018; 15:67.

Zegans ME, Wagner JC, Cady KC, Murphy DM, Hammond JH, O'Toole GA. Interaction between bacteriophage DMS3 and host CRISPR region inhibits group behaviors of Pseudomonas aeruginosa. J Bacteriol. 2009;191:2109.

Zhang X, Cheng C, Sun W, Wang H. Engineering T cells using CRISPR/Cas9 for cancer therapy; in: Methods in Molecular Biology. Humana Press Inc.. 2020:419-33.

Zhen S, Hua L, Takahashi Y, Narita S, Liu YH, Li Y. In vitro and in vivo growth suppression of human papillomavirus 16-positive cervical cancer cells by CRISPR/Cas9. Biochem Biophys Res Commun. 2014;450:1422-6.

Zheng Z, Zhang Y, Liu Z, Dong Z, Xie C, Bravo A, et al. The CRISPR-Cas systems were selectively inactivated during evolution of Bacillus cereus group for adaptation to diverse environments. ISME J. 2020;14:1479-93.

Zhu Y, Klompe SE, Vlot M, van der Oost J, Staals RHJ. Shooting the messenger: RNA-targeting CRISPR-Cas systems. Biosci Rep. 2018;38(3): BSR20170788.

Zuberi A, Misba L, Khan AU. CRISPR interference (CRISPRi) inhibition of luxS gene expression in E. coli: an approach to inhibit biofilm. Front Cell Infect Microbiol. 2017;7:214 